\title{
Interplay between HIV and microRNAs in AIDS-related lymphomas
}

\author{
Giulia De Falco*, Anna Luzzi, Federica Morettini, Anna Onnis, Lorenzo Leoncini \\ From $16^{\text {th }}$ International Symposium on HIV and Emerging Infectious Diseases \\ Marseille, France. 24-26 March 2010
}

\section{Background}

Human immunodeficiency virus (HIV)-induced immune activation of $B$ cells is thought to be a contributing factor to the increased frequency of B-cell malignancies observed in HIV-infected individuals. In some cases, as in Burkitt lymphoma, tumors arise before profound immunosuppression occurs, when the $\mathrm{CD}^{+}$cell count is still high. Therefore, immunodeficiency per se may not be necessary for lymphomagenesis in these patients, and that HIV itself may have an oncogenic potential.

There are no clear answers to explain how HIV leads to malignant transformation, even though several events have been proposed as co-factors in HIV-related tumorigenesis. In particular, the HIV-encoded Tat protein is thought to participate in B-cell abnormalities observed in vivo, as it can be released from the HIV-infected cells and then lead to differential modulation of naïve, memory and germinal center B-cells.

Recent findings indicate a complex interplay between viral proteins and host transcription regulatory machineries, including histone deacetylases (HDACs), histone acetyltransferases (HATs), histone metyltransferases (HMTs) and DNA metyltransferases (DNMTs). Tat can bind to histone acetyltransferases (HATs) p300/CBP, p300/CBP-associated factor, and hGN5. Chromatin remodelling may therefore represent a mechanism of control of gene expression, whose deregulation may eventually lead to malignant transformation.

\section{Methods}

GEP and microRNA profiles were obtained in HIV+ primary tumors.

\section{Results}

We investigated the expression of several chromatin remodelers, both in human cell lines, either transfected or not with Tat, and HIV-positive vs. HIV-negative primary tumors. Gene expression analysis revealed a differential expression of genes involved in chromatin remodelling between HIV-positive and HIV-negative patients. In particular, we observed a modulation of specific acetyltransferases and DNA metyltransferases, which seem to be regulated at the post-transcriptional level.

We then analyzed the expression of microRNAs (miRNAs) predicted to have specific chromatin remodelers as targets, and we observed that these miRNAs resulted significantly down-regulated in HIV-positive subjects.

\section{Discussion}

These findings lead to the hypothesis that HIV may interfere with physiological regulation of cell functions maintained by miRNAs, may be through viral-encoded miRNAs. We are currently analyzing which genetic/epigenetic mechanism underlies HIV-mediated miRNA silencing in host cells.

Collectively, our results support the possible oncogenic role of HIV and open new scenarios in the identification of novel therapeutic targets in HIV-related malignancies.

\section{Published: 11 May 2010}

doi:10.1186/1742-4690-7-S1-P70

Cite this article as: De Falco et al:: Interplay between HIV and

microRNAs in AIDS-related lymphomas. Retrovirology 2010 7(Suppl 1):P70.

\footnotetext{
* Correspondence: defalco@unisi.it

Dept. Human Pathology \& Oncology, University of Siena, Siena, Italy
} 